\title{
Intellectual Property Clearinghouses: The Effects of Reduced Transaction Costs in Licensing*
}

\author{
Reiko Aoki \\ Institute of Economic Research, \\ Hitotsubashi University \\ \& Department of Economics, \\ University of Auckland \\ aokirei@ier.hit-u.ac.jp
}

\author{
Aaron Schiff \\ Department of Economics, \\ University of Auckland \\ a.schiff@auckland.ac.nz
}

July 2008

\begin{abstract}
We focus on downstream uses that combine multiple intellectual property rights and examine the effects of introducing an intellectual property clearinghouse. We identify the two sometimes conflicting function of clearinhouses: transaction costs reduction and coordination. We show that reduction in transaction costs causes licensors to increase royalties in some cases and makes them worse off due to the 'tragedy of the anticommons'. Downstream welfare effects may also be positive or negative and we characterize the effects on downstream manufacturers and final consumers. We also show that total welfare is most likely to increase following a transaction cost reduction when the number of intellectual property rights per downstream use is small, or if rights are relatively substitutable in downstream use, but it is also possible for welfare to decrease.
\end{abstract}

JEL: L24, O34.

Keywords: Intellectual property, licensing, tragedy of the anticommons, clearinghouses.

\footnotetext{
${ }^{*}$ We thank Sadao Nagaoka and participants at the Hitotsubashi Innovation Forum for helpful comments, and we thank the Japan Society for the Promotion of Science for financial support.
} 


\section{Introduction}

A clear trend in intellectual property (IP) is the growth of licensing for downstream uses, as indicated by various studies and surveys such as Razgaitis (2005) and Athreye and Cantwell (2007). The implication is that 'markets for technology' (Arora et al, 2001), where innovations are licensed, are becoming increasingly important in the use and development of IP. This paper examines the effects of introducing a 'clearinghouse' that reduces transaction costs in IP licensing on market efficiency in both a market for technology and downstream markets.

A proliferation of IP rights may result in a 'thicket' (Shapiro, 2001) that can increase costs for development of downstream products that require the use of multiple existing innovations. This issue can arise in high-technology industries such as information technology and biomedicine, as well as content aggregation industries such as radio and television broadcasters and online news providers. To give a specific example, Verbeure et al (2006) describe how genetic diagnostic tests are based on detecting mutations in candidate genes. A single genetic disease can be associated with mutations in many different genes, and an accurate test must check most or all of these. Since gene sequences as well as technologies for amplifying, labeling and detecting sequences can be patented, a new genetic diagnostic test potentially requires licenses to many existing IP rights typically from many different licensors.

There are well-known transaction costs associated with licensing (see, for example, Teece 1977), and these costs are likely to increase with the number of licensing agreements required. In addition, if multiple IP rights are complementary in downstream production, the 'tragedy of the anticommons' (Heller \& Eisenberg, 1998, Buchanan \& Yoon, 2000) may result in license fees that are excessively high. Both the costs of the licensing process and this 'tragedy' may result in lower utilization of IP in terms of downstream use. Due to these issues, a number of 'collective rights' institutions (Merges, 1996) aim to improve efficiency in licensing. Patent pools may help to solve the coordination problem among IP owners that leads to the tragedy of the anticommons. Copyright collectives such as the American Society of Composers and Performers (ASCAP) provide licenses to a bundle of works using standardized contracts and exploit economies of scale in the licensing and monitoring process.

To address the problem of matching IP owners with downstream users, and possibly also solving the tragedy of the anticommons, a number of authors have promoted the idea of IP 'clearinghouses' that act as intermediaries in the licensing process. ${ }^{1}$ As we will discuss, these clearinghouses can potentially provide a range of services in markets for technology, from sim-

\footnotetext{
${ }^{1}$ See van Zimmeren et al (2006), Van Overwalle et al (2006), OECD (2002), and Graff \& Zilberman (2001).
} 
ple searchable databases of available IP, through to packaging and licensing IP on behalf of owners. In Aoki and Schiff (2008) we identify 15 existing third-party clearinghouses (not including copyright collectives). These range from straightforward patent search engines like the Google patent search ${ }^{2}$, to more sophisticated exchange platforms for licensable technologies such as BirchBob $^{3}$ and Yet2.com.

However, there has been little economic analysis of such clearinghouses. In this paper we concentrate on the effects that a clearinghouse might have due to reducing transaction costs on license fees and welfare. We use a simple licensing model in which there is a range of potential uses of existing IP rights that are available for licensing. Each downstream use requires license to a number of IP rights, and these rights may be substitutable to some extent in downstream production. We assume negotiating licenses requires incurring fixed transaction costs, and downstream uses will be exploited if it is profitable for a monopolist to do so. Royalties are set independently by IP owners, so the tragedy of the anticommons occurs in some cases.

An important observation from this model is the role played by the transaction costs in the determination of royalties. Since a manufacturer will not produce anything unless its variable profit can at least cover these costs, the transaction costs can act like a disciplining device on the royalties set by IP owners, and help to offset the tragedy of the anticommons if it occurs. Given this, a reduction in transaction costs by a clearinghouse has three potentially offsetting welfare effects. New products will be produced, and real resource costs of licensing are reduced, but higher royalties flow through into downstream retail prices, which reduces consumption and welfare.

We show that a reduction in transaction costs can make IP owners worse off in aggregate if the substitutability of IP rights is sufficiently low, so that the tragedy of the anticommons causes royalties to rise by a relatively large amount. On the other hand, downstream manufacturers are always better off in aggregate, as the increase in royalties never exceeds the gains that they make from the reduced licensing costs. Consumers may also be worse off, as the increases in per-unit royalties flow through to higher downstream retail prices, and the resulting losses to consumers of existing products can exceed the gains from the introduction of new products. The overall effect on total welfare is ambiguous, with a reduction in licensing costs possibly causing a reduction in equilibrium welfare if substitutability of IP rights is low, if the number of IP rights per downstream product is high, or if the licensing cost reduction is small.

These welfare effects are not spread uniformly across downstream markets. We differentiate downstream markets according to their level of demand. Negative welfare effects can occur in markets with moderate levels of

\footnotetext{
${ }^{2}$ www.google.com/patents

${ }^{3}$ www.birchbob.com
} 
downstream demand, where demand is sufficiently high that production occurs prior to a transaction cost reduction, but where demand is low enough that transaction costs constrain the level of equilibrium royalties. In such markets, equilibrium royalties rise when licensing costs reduce, which can make IP owners worse off if the new royalties are sufficiently high. We show that under some conditions, some IP owners will not want to use a clearinghouse even if it is free.

There is an extensive existing literature on the incentives of innovators to license their IP and optimal licensing contracts. ${ }^{4}$ In this paper we take the licensing decision as given, and focus on the outcome of the licensing process. Within the licensing literature, Arora \& Fosfuri (2003) show, as we do, that reducing transaction costs leads to more licensing, but they do not examine the welfare implications of this. There is also a small economic literature on copyright collectives, which focuses on the effects of these collectives on incentives to create copyrighted works, and the level of royalties set by the collectives. ${ }^{5}$ Similarly, the literature on patent pools examines the centralized royalties set by a pool and the incentives for pool formation. ${ }^{6} \mathrm{In}$ contrast, we focus on the effects of transaction costs on decentralized licensing. Finally, Aoki (2006) considers IP clearinghouses as network providers and examines the demand for and stability of these systems in contrast with patent pools, while in this paper we focus on general effects of transaction cost reductions in a market for technologies.

The organization of the rest of this paper is as follows. The next section briefly describes IP clearinghouses and their role in the licensing process. ${ }^{7}$ Section 3 presents a simple licensing model and section 4 characterizes the equilibrium of the model. Then section 5 examines what happens in equilibrium when transaction costs are reduced. Section 6 offers concluding comments and suggestions for future work.

\section{Intellectual Property Clearinghouses}

Clearinghouses act like intermediaries in the licensing process. For example, van Zimmeren et al (2006) promote the idea of establishing a clearinghouse of biotechnology IP, to assist the development of new genetic diagnostic tests and other advances that require licenses to multiple existing IP rights. A concrete example of a third-party clearinghouse is Yet2.com. It was founded in 1999 with joint investment from Siemens, Bayer, Honeywell, Dupont, Procter \& Gamble, Caterpillar, and NTT Leasing. It describes itself as a 'technology marketplace' and provides an online platform where technologies

\footnotetext{
${ }^{4}$ For example, Gallini and Wright (1990) and Katz and Shapiro (1985).

${ }^{5}$ Besen et al (1992), Kleit (2000), Hollander (1984).

${ }^{6}$ See, for example, Lerner \& Tirole (2004) and Aoki \& Nagaoka (2005).

${ }^{7}$ More detailed descriptions and discussion are contained in Aoki \& Schiff (2007).
} 


\begin{tabular}{|c|c|c|c|}
\hline \multirow{2}{*}{ 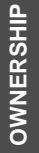 } & $3^{\text {rd }}$ Party & I & II \\
\hline & Collective & III & IV \\
\hline & & $\begin{array}{l}\text { Informational } \\
\text { Clearinghouse }\end{array}$ & $\begin{array}{c}\text { Licensing } \\
\text { Clearinghouse }\end{array}$ \\
\hline & & $\begin{array}{l}\text { Information } \\
\text { Only }\end{array}$ & $\begin{array}{c}\text { Information \& } \\
\text { Licensing }\end{array}$ \\
\hline & & FUN & IION \\
\hline
\end{tabular}

Figure 1: Classification of clearinghouses (from Aoki \& Schiff, 2007).

that available for licensing can be listed and searched.

Yet2.com operates independently and raises revenue from both potential licensors and licensees. Potential licensees can perform basic searches of listed technologies for free, but more advanced searching and viewing complete details of listings requires purchasing a subscription. Listing technologies also requires a subscription, and Yet2.com charges a commission on any successful licensing arrangement made as a result of its services.

The potential functions of clearinghouses are discussed by van Zimmeren et al (2006). These range from simply providing information about existing IP through to issuing standardized licenses and collecting royalties on behalf of IP owners. In our view, the most important functional distinction is whether or not the clearinghouse sells licenses on behalf of IP owners. This is because centralization of licensing is necessary to solve the coordination problem that leads to the tragedy of the anticommons, if it occurs. Another important distinction is whether the clearinghouse exists as an independent third-party or operates on a collective basis on behalf of IP owners, as this will affect its incentives. In Aoki \& Schiff (2007) we therefore propose a broad classification of clearinghouses into four groups, as shown in Figure 1. Within this classification, Yet2.com is a third-party informational clearinghouse (type I), and copyright collectives such as ASCAP are collective licensing clearinghouses (type IV). We are not aware of any type II or III clearinghouses that cover a broad range of IP, although some patent pools for specific technologies are administered by third-parties (type II). ${ }^{8}$ Types II and IV could be considered as collective rights organizations (Merges, 1996).

The functional dimension of this classification makes clear the two possible roles of a clearinghouse: coordination and transaction cost reduction. In this paper we focus on the latter. ${ }^{9}$ There are a number of ways that

\footnotetext{
${ }^{8}$ Aoki \& Nagaoka (2005) describe the operation of several existing patent pools.

${ }^{9}$ The basic coordination issue is the same as that dealt with in the literature on patent pools. See, for example, Lerner \& Tirole (2004).
} 
a clearinghouse may be able to reduce transaction costs. First, a clearinghouse can spread the costs of the platform used for searching and identifying existing IP rights over its user base. As a result, it may also be economic for it to deploy a more sophisticated search technology than individual users could implement. Second, a clearinghouse may be able to apply technological and legal expertise to assist with licensing and reduce the complexities of the process. Third, if a clearinghouse conducts licensing on behalf of IP owners, it may be able to exploit economies of scale in this process, as certain elements of the contracts and negotiations may be common across technologies.

\section{The Model}

There is a continuum of downstream products that can be produced. Products are independent of each other and differ by the level of their demand. Each product has a linear demand and the intercept differs across products, so demand for a particular product is given by

$$
p=\alpha-q,
$$

where $p$ is the retail price for this product and $q$ is the quantity sold. The parameter $\alpha$ differs by product and is distributed uniformly on $[0, A]$.

Each downstream product is manufactured by an independent monopolist. ${ }^{10}$ Each monopolist requires licenses from $n=1,2,3, \ldots$ independent upstream IP owners. These $n$ IP rights are specific to a single downstream use. ${ }^{11}$ For a given downstream market, let $x_{i}$ be the 'quantity' of IP right $i$ that is used. Each monopolist has a symmetric CES production technology, so that

$$
q=\left(\sum_{i=1}^{n} x_{i}^{\beta}\right)^{1 / \beta}
$$

where $\beta \in(-\infty, 0) \cup(0,1]$. This formulation allows us to present a very general analysis regarding technology substitutability. If $\beta=1$, IP rights are perfect substitutes and in the limit as $\beta \rightarrow-\infty$ they are perfect complements. We assume $n$ and $\beta$ are the same for all downstream markets. ${ }^{12}$

\footnotetext{
${ }^{10}$ Within our model, a downstream monopoly is the optimal licensing structure for the IP owners.

${ }^{11}$ Alternatively, if IP rights have multiple downstream uses, we assume that an IP owner sets royalties for each downstream use independently.

${ }^{12}$ Without this assumption, we would need to make additional assumptions about the distribution of $n$ and $\beta$ across downstream markets. We cannot see any basis for any particular relationship between $\alpha, \beta$ and $n$, so such assumptions would necessarily be arbitrary and would complicate the analysis.
} 
For a given downstream market, each of the $n$ independent IP owners charges a per-unit royalty $r_{i}$. For what follows, it will be useful to define

$$
z=\sum_{i=1}^{n} r_{i}^{\frac{\beta}{\beta-1}} .
$$

Aside from royalties, we assume that manufacturers have no other variable costs. With the CES production function, the solution to a manufacturer's cost minimization problem gives a total variable cost of $c(q)=z^{\frac{\beta-1}{\beta}} q$.

Each manufacturer must also pay a fixed transaction cost of $s(n) \geq 0$ to identify and locate IP owners and negotiate licenses with them. ${ }^{13}$ This cost is not sunk until the licensing process is initiated. We assume that $s(n)$ is increasing in $n$ and $s(0)=0$. A manufacturer's profit in a given downstream market is therefore

$$
\pi_{M}=\left(\alpha-q-z^{\frac{\beta-1}{\beta}}\right) q-s(n) .
$$

We will assume that manufacturers in at least some markets make strictly positive profits in equilibrium. This will require that demand in some markets is sufficiently high, and in particular we assume that

$$
A>\frac{2(2-(1+n) \beta)}{1-\beta} \sqrt{s(n)} .
$$

Upstream, the $n$ IP owners for each downstream market set royalties simultaneously and independently to maximize their royalty revenues. The timing is that IP owners set and announce royalties first. The downstream monopolists then take these royalties as given and choose whether or not to incur $s(n)$, and if so, the quantity of output to produce.

\section{Equilibrium}

Under our assumptions the downstream markets are completely independent. We characterize the equilibrium for a given $\alpha$ by first solving the downstream manufacturer's profit maximization problem taking the royalties as given. We then solve the simultaneous game among the $n$ IP owners for this market to determine the equilibrium royalties.

Given the downstream profit function (1), if production of a given product is profitable, the manufacturer produces

$$
q_{M}=\frac{1}{2}\left(\alpha-z^{\frac{\beta-1}{\beta}}\right)
$$

\footnotetext{
${ }^{13}$ We assume all fixed licensing costs are borne by downstream manufacturers and not IP owners. This is because our analysis starts after IP owners have already commited to license their IP, and thus making them bear some part of the licensing costs will not change their behavior in the model. As long as downstream manufacturers incur some proportion of the licensing costs, the basic qualitative results of our model will continue to hold.
} 
and makes profit

$$
\pi_{M}=\frac{1}{4}\left(\alpha-z^{\frac{\beta-1}{\beta}}\right)^{2}-s(n) .
$$

Thus for production to be profitable we must have

$$
\alpha \geq z^{\frac{\beta-1}{\beta}}+2 \sqrt{s(n)},
$$

otherwise the manufacturer will choose not to incur the fixed cost $s(n)$ and will produce nothing. Note that even if all royalties are zero for this product, production is not profitable unless $\alpha \geq 2 \sqrt{s(n)}$. The presence of the transaction costs restricts the set of products that are produced, regardless of royalties. We thus define $\underline{\alpha}=2 \sqrt{s(n)}$.

If the manufacturer does produce some quantity $q>0$, given the CES production function, the demand for licenses from IP right $i$ is $x_{i}=\phi_{i} q$, where $\phi_{i}=r_{i}^{\frac{1}{\beta-1}} z^{-\frac{1}{\beta}}$. Thus we have

$$
x_{i}=\left\{\begin{array}{ll}
\phi_{i} q_{M} & \text { for } z^{\frac{\beta-1}{\beta}} \leq \alpha-2 \sqrt{s(n)} \\
0 & \text { otherwise }
\end{array} .\right.
$$

The term $\phi_{i}$ captures the possibilities for substitution of IP rights. Demand for IP right $i$ always decreases in $r_{i}$ due to manufacturer substitution towards other IP rights $\left(\phi_{i}\right.$ reduces), and due to a reduction in production as the marginal cost increases ( $q_{M}$ reduces). On the other hand, across IP rights, $x_{i}$ may increase or decrease in response to an increase in $r_{j}$, since these substitution and quantity effects work in opposite directions.

Upstream, the royalty revenues of IP owner $i$ are

$$
R_{i}=x_{i} r_{i}
$$

Given the symmetry of our setup, we concentrate on symmetric equilibria where $r_{i}=r^{*}$ for all $i$, for a given $\alpha$. The following result is useful for characterizing the equilibria.

Lemma 1 If $\beta>1 / n$, at any symmetric royalty $r>0$ where downstream production is profitable, any individual IP owner has an incentive to undercut, that is, $R_{i}$ is maximized for $r_{i}<r$.

Proof. In the Appendix.

Intuitively, when $\beta$ is high, IP rights are relatively substitutable. At a symmetric royalty, when $\beta>1 / n$, substitution between IP rights by manufacturers is so strong that a unilateral royalty reduction is always profitable for any individual IP owner. This drives equilibrium royalties to zero in 
all markets. The following proposition characterizes the symmetric pure strategy equilibrium royalties. ${ }^{14}$

Proposition 1 For $1 / n<\beta \leq 1$, symmetric equilibrium royalties are zero. For $\beta \leq 1 / n$ and $\beta \neq 0$, there is a symmetric pure strategy equilibrium with

$$
r^{*}(\alpha)= \begin{cases}\frac{n^{\frac{1}{\beta}}\left(\frac{1}{n}-\beta\right) \alpha}{2-(1+n) \beta} & \text { for } \bar{\alpha} \leq \alpha \leq A \\ n^{\frac{1-\beta}{\beta}}(\alpha-2 \sqrt{s(n)}) & \text { for } \underline{\alpha} \leq \alpha \leq \bar{\alpha}\end{cases}
$$

where

$$
\bar{\alpha}=\frac{2(2-(1+n) \beta)}{1-\beta} \sqrt{s(n)}
$$

Proof. In the Appendix.

If $\beta \leq 1 / n$, equilibrium royalties are unconstrained by downstream manufacturer profitability if demand $(\alpha)$ is sufficiently high. ${ }^{15}$ Otherwise, equilibrium royalties in markets for $\underline{\alpha} \leq \alpha \leq \bar{\alpha}$ are constrained to the level at which the manufacturer just breaks even. Observe that $r^{*}(\alpha)$ is an increasing piecewise linear function of $\alpha$ and it is straightforward to verify that it is continuous in $\alpha$. From now on we will assume that $\beta \leq 1 / n$, as a change in transaction costs will not affect the equilibrium if $\beta>1 / n$.

In this model, the 'tragedy of the anticommons' occurs for a given downstream market if the equilibrium royalty exceeds the level that maximizes the royalty revenues of the IP owners in that market. Let $\widetilde{r}$ denote the symmetric jointly optimal royalty for a given $\alpha$. To characterize $\widetilde{r}$, at a symmetric royalty $r$, from (5) the demand for each IP right becomes a downward-sloping linear function of $r$ :

$$
x(r)=\frac{1}{2} n^{-\frac{1}{\beta}}\left(\alpha-n^{\frac{\beta-1}{\beta}} r\right) .
$$

The total royalty revenues of the $n$ IP owners is $R=n x(r) r$, so the firstorder condition is

$$
\frac{\partial R}{\partial r}=\frac{1}{2} n^{1-\frac{1}{\beta}}\left(\alpha-2 n^{\frac{\beta-1}{\beta}} r\right)=0
$$

which gives

$$
\widetilde{r}=\frac{1}{2} n^{\frac{1-\beta}{\beta}} \alpha .
$$

\footnotetext{
${ }^{14}$ There are also uninteresting symmetric equilibria that we ignore. For example, if $r_{i}=$ $r$ for all $i$ and $r$ is very large, downstream production will not occur, but no IP owner will be able to make production profitable by unilaterally reducing $r_{i}$, thus $r$ is an equilibrium, and there will be a multiplicity of such equilibria. In addition, when the downstream profitability constraint binds in equilibrium, there may be multiple asymmetric equilibria that satisfy this constraint, which we also ignore.

${ }^{15}$ Our earlier assumption (2) guarantees that $A>\bar{\alpha}$.
} 

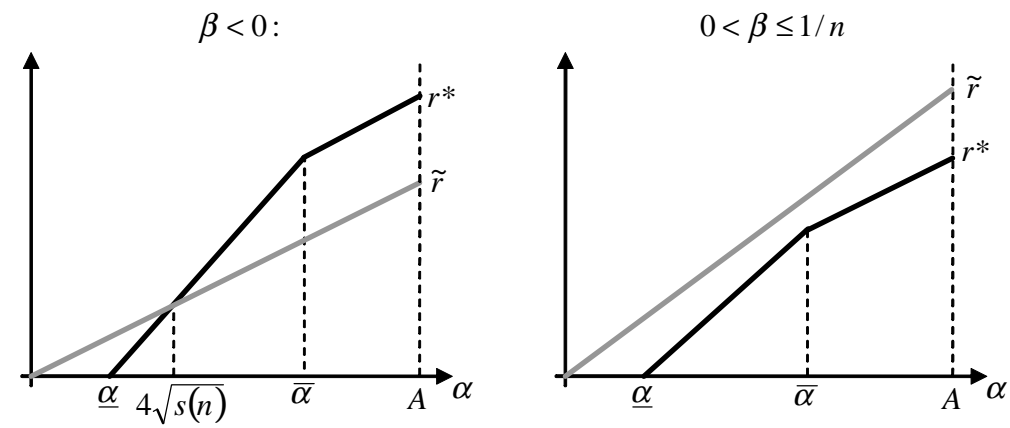

Figure 2: Equilibrium $\left(r^{*}\right)$ and unconstrained jointly optimal $(\widetilde{r})$ royalties as a function of the demand intercept $(\alpha)$ assuming $n \geq 2$.

Let us define $\rho(n)=r^{*} / \widetilde{r}$ as the ratio of the equilibrium royalty to the (unconstrained) joint optimum for a given $n$, so the tragedy of the anticommons occurs if $\rho(n)>1$. From (7) and (9) we have

$$
\rho(n)= \begin{cases}\frac{2(1-n \beta)}{2-(1+n) \beta} & \text { for } \bar{\alpha} \leq \alpha \leq A \\ 2-4 \sqrt{s(n)} / \alpha & \text { for } \underline{\alpha} \leq \alpha \leq \bar{\alpha}\end{cases}
$$

Proposition 2 The tragedy of the anticommons occurs for a given downstream market if $n \geq 2, \beta<0$ and $\alpha>4 \sqrt{s(n)}$. Otherwise, the equilibrium royalty in that market is lower than the joint optimum.

Proof. In the Appendix.

Intuitively, the equilibrium royalty exceeds the joint optimum if substitutability of IP rights is low, there are at least two licensors per downstream market, and the downstream profitability constraint is not very severe. ${ }^{16}$ Figure 2 illustrates $r^{*}$ and $\widetilde{r}$ for different products, depending on the value of $\beta$, assuming that $n \geq 2$.

It is also interesting to investigate how changes in the number of IP rights per downstream market and the substitutability of rights affect the ratio $\rho$. From (10) we have

$$
\rho(n+1)-\rho(n)=\left\{\begin{array}{ll}
-\frac{2(1-\beta) \beta}{(2-\beta(1+n))(2-\beta(2+n))} & \text { for } \bar{\alpha} \leq \alpha \leq A \\
\frac{4}{\alpha(\sqrt{s(n)}-\sqrt{s(n+1)})} & \text { for } \underline{\alpha} \leq \alpha \leq \bar{\alpha}
\end{array},\right.
$$

\footnotetext{
${ }^{16}$ These results also indicate that joint setting of royalties by a patent pool will not be welfare enhancing for a given downstream market when patents are sufficiently substitutable $(\beta>0)$, while it may or may not enhance welfare when patents are less substitutable $(\beta<0)$. Similar results are obtained in a different framework by Lerner and Tirole (2004)
} 
and also

$$
\frac{\partial \rho}{\partial \beta}= \begin{cases}-\frac{2(n-1)}{(\beta+n \beta-2)^{2}} & \text { for } \bar{\alpha} \leq \alpha \leq A \\ 0 & \text { for } \underline{\alpha} \leq \alpha \leq \bar{\alpha}\end{cases}
$$

which give the following proposition.

Proposition 3 When the downstream profitability constraint does not bind $(\bar{\alpha} \leq \alpha \leq A)$, adding an additional IP right causes $\rho$ to increase when $\beta<0$ and to decrease when $\beta>0$, while increasing the substitutability of rights decreases $\rho$. If the downstream profitability constraint binds $(\underline{\alpha} \leq \alpha \leq \bar{\alpha}), \rho$ decreases when $n$ increases under the assumption that $s(n)$ is an increasing function, and is independent of $\beta$.

Proposition 3 says that if downstream profitability is not a factor in equilibrium, then an increase in $n$ increases the ratio of equilibrium royalties to the joint optimum when $\beta<0$, while the opposite occurs when $\beta>0$. In either case, the equilibrium royalty moves further away from the joint optimum, thus IP owners are always hurt by an increase in $n$, but the mechanism differs depending on whether the tragedy of the anticommons occurs or not. If $\beta<0$, the tragedy of the anticommons occurs in these markets, and adding another IP right only makes it worse. On the other hand, if $\beta>0$, the tragedy does not occur and adding another IP right stimulates competition among IP owners in the sense that royalties fall relative to the joint optimum. For markets that are profit constrained, regardless of the value of $\beta$, increasing $n$ reduces equilibrium royalties, as the downstream profit constraint becomes more severe. If the tragedy of the anticommons occurs in such markets, this can make IP owners better off, as a more severe constraint may bring royalties closer to the joint optimum.

\section{Effect of a Transaction Cost Reduction}

In this section we examine the effects on the equilibrium of the model of reducing transaction costs from $s_{0}(n)$ to $s_{1}(n)<s_{0}(n)$ for all $n$. Let us define $\underline{\alpha}_{0}$ and $\underline{\alpha}_{1}$ as the respective values of $\underline{\alpha}$ given $s_{0}(n)$ and $s_{1}(n)$, and similarly define $\bar{\alpha}_{0}$ and $\bar{\alpha}_{1}$ as the respective values of $\bar{\alpha}$. Clearly, $\underline{\alpha}_{1}<\underline{\alpha}_{0}$ and $\bar{\alpha}_{1}<\bar{\alpha}_{0}$.

In general, the effects of a transaction cost reduction differ across downstream markets. Some products that were not produced will become profitable. In addition, the set of products where equilibrium royalties are constrained by downstream profitability shrinks, (i.e., $\bar{\alpha}_{1}-\underline{\alpha}_{1}<\bar{\alpha}_{0}-\underline{\alpha}_{0}$ ) and the profitability constraint relaxes in the markets that remain constrained. This affects equilibrium royalties and hence retail prices in some markets. The resource costs of licensing also reduce. In what follows, we discuss the implications of these effects for consumers, downstream manufacturers, IP owners, and total welfare. 

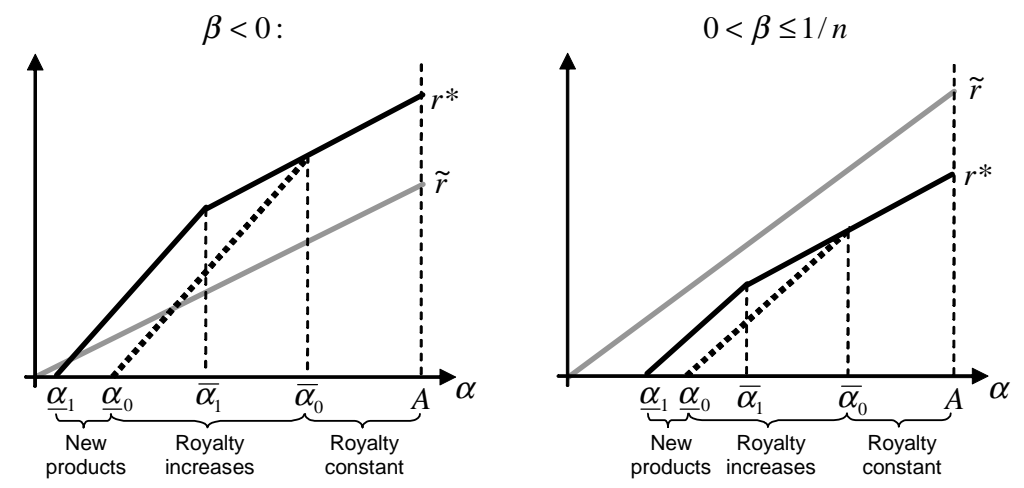

Figure 3: Effects on equilibrium royalties of a transaction cost reduction, assuming that $n \geq 2$ and $\bar{\alpha}_{1}>\underline{\alpha}_{0}$. The dotted line shows the equilibrium royalties for profit-constrained products at $s_{0}(n)$ and the solid black line shows the equilibrium royalties at $s_{1}(n)$ as well as the equilibrium royalties for unconstrained products at $s_{0}(n)$.

First, consider the effect on equilibrium royalties. From (7) it is clear that equilibrium royalties are unchanged for products where the original equilibrium was not constrained by downstream profitability $\left(\alpha \geq \bar{\alpha}_{0}\right)$. The exact change in royalties in other markets depends on the size of the transaction cost reduction. If the cost reduction is large enough, all products that were profit constrained at $s_{0}(n)$ become unconstrained at $s_{1}(n)$, that is, $\bar{\alpha}_{1}<\underline{\alpha}_{0}$. For smaller cost reductions, some of these products remain profit constrained at $s_{1}(n)$ and $\underline{\alpha}_{0}<\bar{\alpha}_{1}<\bar{\alpha}_{0}$. However, in either case, the royalties for all products that were constrained at the original transaction $\operatorname{cost}\left(\underline{\alpha}_{0} \leq \alpha \leq \bar{\alpha}_{0}\right)$ increase, because equilibrium royalties for constrained products are negatively related to $s(n)$, and unconstrained equilibrium royalties always exceed the constrained equilibrium level for a given $\alpha$. Finally, products in $\underline{\alpha}_{1} \leq \alpha \leq \underline{\alpha}_{0}$ were not produced in equilibrium at $s_{0}(n)$, but will be produced at $s_{1}(n)$ at a positive royalty level. The following proposition summarizes this analysis.

Proposition $4 A$ transaction cost reduction from $s_{0}(n)$ to $s_{1}(n)$ leaves equilibrium royalties unchanged for products that were not profit constrained at $s_{0}(n)\left(\alpha \geq \bar{\alpha}_{0}\right)$, equilibrium royalties increase in markets that were profitconstrained at $s_{0}(n)\left(\underline{\alpha}_{0} \leq \alpha \leq \bar{\alpha}_{0}\right)$, and new products in $\underline{\alpha}_{1} \leq \alpha \leq \underline{\alpha}_{0}$ are produced at positive royalties.

Figure 3 illustrates Proposition 4. The impact on equilibrium royalties relative to the joint optimum royalty depends on $\beta$. From Proposition 2 , if $\beta>0$, equilibrium royalties never exceed the joint optimum, thus reducing transaction costs never harms IP owners in this case because it only takes 
them closer to the joint optimum. On the other hand, if $\beta<0$, equilibrium royalties may exceed the joint optimum and a transaction cost reduction will make IP owners worse off in some markets. IP owners in profit-constrained markets where the equilibrium royalty at $s_{0}(n)$ already exceeds the joint optimum $\left(4 \sqrt{s_{0}(n)} \leq \alpha \leq \bar{\alpha}_{0}\right)$ will be made worse off as equilibrium royalties increase further beyond the optimum. In addition, some IP owners in profit-constrained markets where the original equilibrium royalty was below the joint optimum may be made worse off, if the equilibrium royalties increase sufficiently far beyond the joint optimum.

Due to these effects, when $\beta<0$, it is possible that the aggregate welfare of IP owners (measured by total royalty revenues) may decrease following a transaction cost reduction. This occurs if equilibrium royalties increase beyond the joint optimum by a sufficiently large amount in sufficiently many markets that the harm in these markets exceeds the benefits in the markets where equilibrium royalties remain below the joint optimum plus the benefits from the production of new products. In particular, the total royalty revenues of all IP owners is given by

$$
T R=\int_{\underline{\alpha}}^{A} \frac{n \times r^{*}(\alpha) \times x\left(r^{*}(\alpha)\right)}{A} d \alpha .
$$

Evaluating (11) yields the following result.

Proposition 5 In aggregate, a transaction cost reduction increases total royalty revenues if $(2-n) \beta<1$. This is true for any feasible $\beta$ if $n \leq 2$. If $n>2$, it requires $\beta>1 /(2-n)$. Otherwise, total royalty revenues decrease.

Proof. In the Appendix.

This result says that the aggregate effects of a transaction cost reduction on IP owners depends on the substitutability among IP rights relative to their number. Provided that IP rights are sufficiently substitutable, IP owners are not made worse off in aggregate, as the negative effects (if any) from a worsening of the tragedy of the anticommons are relatively small. This is always true for $n \leq 2$ or $\beta>0$. If $n>2$, the range of negative values of $\beta$ in which IP owners are better off shrinks as $n$ increases. Recall from Proposition 3 that an increase in $n$ increases the ratio of equilibrium royalties to the joint optimum when $\beta<0$, while increasing $\beta$ reduces the ratio in unconstrained markets. Thus when $n$ is higher, to generate an aggregate improvement in the welfare of IP owners from a transaction cost reduction, the substitutability of IP rights must also be higher.

Next consider the effects on downstream manufacturers. From Proposition 4 , manufacturers of products that were not profit-constrained in equilibrium at $s_{0}(n)\left(\alpha \geq \bar{\alpha}_{0}\right)$ are better off by the amount of $s_{0}(n)-s_{1}(n)$, as the royalties that they face are unchanged while their transaction costs 
decrease. Manufacturers of products that are profit-constrained in equilibrium at $s_{1}(n)\left(\underline{\alpha}_{1} \leq \alpha \leq \bar{\alpha}_{1}\right)$ are indifferent to a transaction cost reduction. All such manufacturers were either profit-constrained or did not produce anything at $s_{0}(n)$, so they make zero profits both $s_{0}(n)$ and $s_{1}(n)$. Finally, some products that were profit-constrained in equilibrium at $s_{0}(n)$ will become unconstrained at $s_{1}(n)$ (for $\bar{\alpha}_{1} \leq \alpha \leq \bar{\alpha}_{0}$ ). Thus the equilibrium profits for such manufacturers become strictly positive. Overall, since no individual manufacturers are worse off and some are better off, aggregate manufacturer profit increases. The following proposition summarizes these results.

Proposition 6 In aggregate, manufacturers are always better off from a transaction cost reduction. Individually, those in markets that are profitconstrained in equilibrium at $s_{1}(n)$ are indifferent, while those in markets that are unconstrained in equilibrium at $s_{1}(n)$ are better off.

Next let us consider the effect on consumers as measured by consumer surplus in the downstream markets. Consumers of new products $\left(\underline{\alpha}_{1} \leq\right.$ $\left.\alpha \leq \underline{\alpha}_{0}\right)$ are better off. Consumers in markets that were profit-constrained in equilibrium at $s_{0}(n)\left(\underline{\alpha}_{0} \leq \alpha \leq \bar{\alpha}_{0}\right)$ are made worse off because the higher equilibrium royalties in such markets result in higher retail prices. Consumers of products that were unconstrained in equilibrium at $s_{0}(n)$ $\left(\alpha \geq \bar{\alpha}_{0}\right)$ are indifferent, as the royalties and hence retail prices for such products remain unchanged.

The overall effect on consumers depends on the tradeoff between the positive effect of the introduction of new products, with the negative effect of higher retail prices in some markets. Aggregate consumer surplus is

$$
C S=\int_{\underline{\alpha}}^{A} \frac{\frac{1}{2} q_{M}\left(r^{*}(\alpha)\right)^{2}}{A} d \alpha .
$$

Evaluating (12) and and examining how it changes with $s(n)$, together with the above discussion gives the following result.

Proposition 7 In aggregate, consumers are better off from a transaction cost reduction if $\beta>1 /(2 n-1)$, otherwise they are worse off. Individually, consumers in markets that were profit-constrained at $s_{0}(n)$ are worse off, those in unconstrained markets at $s_{0}(n)$ are indifferent, and consumers of new products are better off.

Proof. In the Appendix.

This result says that provided IP rights are sufficiently substitutable relative to $n$, consumers in aggregate are better off from a transaction cost reduction. When IP rights are highly substitutable, the increases in equilibrium royalties and hence retail prices in profit-constrained markets never 
outweigh the gains from introducing new products. However, the requirement on substitutability is strong, since the minimum value of $\beta$ at which consumers are better off, $1 /(2 n-1)$, is always greater than zero.

In terms of total welfare, there are three basic effects of a reduction in $s(n)$. The first is the direct effect that transaction costs of manufacturers are reduced, which raises welfare. Second, royalties increase in some markets, which reduces consumption and welfare in these markets. Third, some new products are introduced, which increases welfare. The overall impact on welfare is the balance of these three effects.

Proposition 8 Equilibrium total welfare increases for any transaction cost reduction if

$$
s_{0}(n) \leq \frac{4 A^{2}(1-\beta)^{4}}{\left((2 n(n+1)+3) \beta^{2}-(8+6 n) \beta+7\right)^{2}} .
$$

In addition, if

$$
\frac{n-2-\sqrt{3}(n-1)}{2 n^{2}-2 n-1} \leq \beta<\frac{1}{n}
$$

then any parameter values that satisfy the assumption (2) also satisfy (13). Otherwise, if (13) does not hold, the transaction cost reduction must be sufficiently large to increase welfare. The required transaction cost reduction increases with $n$ and decreases with $\beta$.

Proof. In the Appendix.

This result says that if IP rights are sufficiently substitutable relative to $n$, any transaction cost reduction, no matter how small, will improve welfare. In such a case, the welfare losses due to increases in royalties in constrained markets are never so large that they outweigh the positive effects of the introduction of new products and the reduced resource costs of licensing. Alternatively, if the initial transaction cost $s_{0}(n)$ is low enough, the negative effects of higher equilibrium royalties from a transaction cost reduction will be confined to relatively few profit-constrained markets, and will not outweigh the positive welfare effects. ${ }^{17}$

Otherwise, if neither of these two conditions are satisfied, the transaction cost reduction must be large enough so that the positive effects outweigh the negative effects of higher equilibrium royalties in constrained markets. Since it is not possible to reduce transaction costs below $s(n)=0$, it may be the case that eliminating transaction costs will not improve welfare. If this

${ }^{17}$ The proportion of markets that are profit-constrained is

$$
\frac{\bar{\alpha}-\underline{\alpha}}{A}=\frac{2(1-n \beta)}{A(1-\beta)} \sqrt{s(n)}
$$

which shrinks as $s(n)$ reduces because $\beta \leq 1 / n$. 


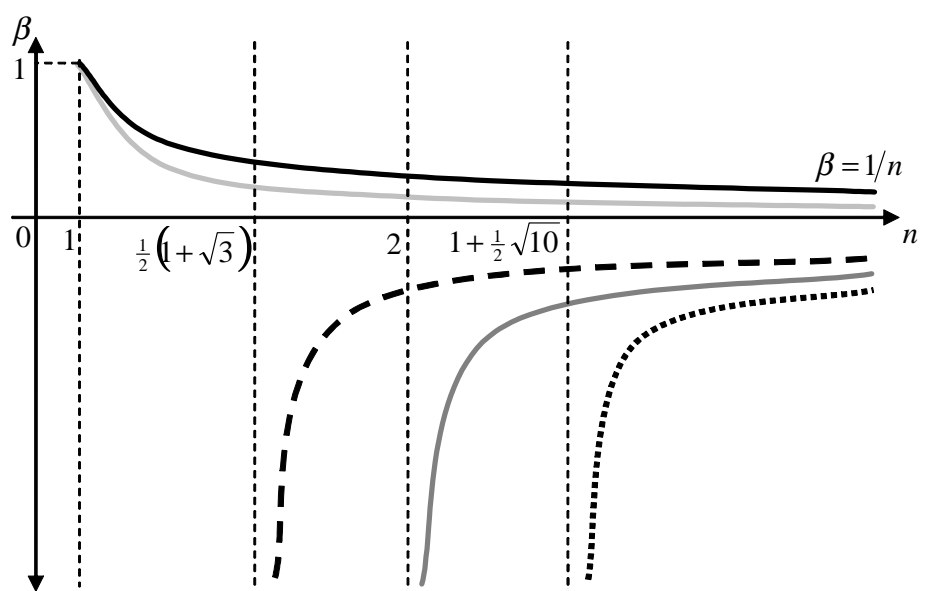

Figure 4: A comparison of the parameter regions implied by the aggregate welfare results (not to scale). Above the light grey line, a transaction cost reduction makes consumers better off. Above the dashed line, total welfare is guaranteed to improve. Between the dashed and dotted lines, total welfare improves if the transaction cost reduction is sufficiently large. Below the dotted line, total welfare may not improve even if transaction costs are eliminated. Above the dark grey line, IP owners are better off.

occurs it implies that the tragedy of the anticommons is sufficiently prevalent that the disciplining effect of transaction costs on equilibrium royalties is the most important factor in the total welfare effects.

Corollary 1 If $\beta<-(5+\sqrt{10}(n-1)) /\left(2 n^{2}-4 n-3\right)$ there exist parameter values that satisfy $\beta \leq 1 / n$ and (2) such that welfare never improves from any transaction cost reduction.

Proof. In the Appendix.

Figure 4 compares the parameter regions obtained in the aggregate welfare results, taking $n$ as continuous for simplicity. If substitutability of IP rights is very high, a transaction cost reduction makes consumers better off (above the light grey line). Total welfare is guaranteed to improve at sufficiently high negative values of $\beta$ (above the dashed line), while lower values require a large enough transaction cost reduction to improve welfare (between the dashed and dotted lines). If substitutability is very low (below the dotted line), total welfare may not improve even if transaction costs are eliminated. IP owners are better off for intermediate values of substitutability (above the dark grey line).

Although the particular results shown in Figure 4 are driven by our particular assumptions such as linear downstream demand and a uniform distribution of demand intercepts, these results demonstrate the tension between 


\begin{tabular}{|c|c|c|c|c|}
\hline Royalties & & 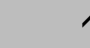 & & - \\
\hline IP owners $(\beta<0)$ & $\uparrow$ & $\uparrow / \downarrow$ & $\downarrow$ & - \\
\hline IP owners $(\beta>0)$ & & 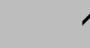 & & - \\
\hline Manufacturers & & - & & \\
\hline Consumers & $\uparrow$ & & $\downarrow$ & - \\
\hline Welfare & $\uparrow$ & & $\uparrow / \downarrow$ & $\uparrow$ \\
\hline
\end{tabular}

Figure 5: Summary of effects on royalties and welfare in downstream markets of a transaction cost reduction where $\bar{\alpha}_{1}>\underline{\alpha}_{0}$.

the total welfare effects and the effects on consumers and IP owners. Consumers are easily made worse off by a transaction cost reduction as royalty increases can span many markets, while the introduction of new products will necessarily be in low demand and hence low consumer surplus markets. Thus a transaction cost reduction that improves overall welfare may make consumers worse off, independent of who pays the transaction costs. In addition, it is possible that IP owners are made better off while total welfare does not improve, if substitutability is moderately low, so that royalty increases cause sufficiently much harm to consumers, but the increases are not so large that the aggregate effects on IP owners are negative.

Finally, as discussed above, the effects of a transaction cost reduction differ substantially across downstream markets. Figure 5 summarizes these effects assuming that $\bar{\alpha}_{1}>\underline{\alpha}_{0}$. In general, the incentives of individual manufacturers and IP owners to use a cost-reducing clearinghouse differ from each other and from total welfare. While manufacturers are never made worse off, the same can be said for IP owners only if IP rights are relatively substitutable, so that $\beta>0$. If $\beta<0$, IP owners may be worse off in markets with intermediate values of $\alpha$.

These results have implications for the operation of a clearinghouse business. A clearinghouse will need to attract both manufacturers and IP owners to use its services. However, as Figure 5 shows, the incentives of manufacturers and IP owners to join a clearinghouse are not always aligned. Manufacturers will always be (weakly) willing to join a clearinghouse, although manufacturers of relatively low demand products may not be willing to pay a price to do so. The demand by IP owners depends on the substitutability of IP rights relative to their number, and downstream demand. If substitutability is low, only IP owners in relatively low demand markets will be willing to pay a price to join a clearinghouse, while IP owners in medium demand markets may not be willing to join even if it is free. On the other 
hand, if substitutability is high enough, all IP owners except those in relatively high demand markets will be willing to pay to join.

\section{Conclusion}

In this paper we have used a simple model of IP licensing to examine the effects of a reduction in costs associated with the licensing process on royalties and welfare. Our motivation was a desire to understand how IP clearinghouses can affect markets for technology. We showed that noncooperative royalty setting by IP owners can lead to the tragedy of the anticommons, and that considerations of downstream profitability can be a disciplining device to offset this. Therefore, a reduction in licensing costs can lead to negative welfare effects that arise from higher equilibrium royalties, and this can make IP owners and consumers worse off, and in some cases can be sufficiently large to offset the gains from the introduction of new downstream products and reduced costs for manufacturers.

While we have used a model with fairly specific assumptions in this paper, we believe the qualitative results are reasonably robust to changes in the particular assumptions. The basic results come from two sources. First, IP owners have overlapping claims to a downstream product, and this results in a tendency to set excessive royalties if substitutability of rights is sufficiently low, and this is exacerbated if there are more IP rights per downstream market. Second, there are transaction costs in licensing that bear on the downstream decision to obtain licenses, and which can affect the upstream strategic behavior of IP owners. These two things underpin the basic intuition developed in this paper, and similar effects could be obtained in alternative models with different assumptions.

Our analysis was confined to modeling a reduction in fixed licensing costs that applied to all negotiations. We believe this is a useful first step in understanding the implications of IP clearinghouses for markets for technology, as it characterizes the full extent of the potential welfare implications of an informational clearinghouse. An interesting next step for future research would be to characterize the demand by manufacturers and IP owners that a clearinghouse would face for its services. As we have seen, the welfare effects on IP owners are complex, and some IP owners may be unwilling to join a clearinghouse even if its services are provided for free. It would be useful to understand the profit-maximizing behavior of a third-party clearinghouse, and the implications for welfare.

We have also maintained the assumption that royalties are set independently by IP owners. In patent pools and copyright collectives, and under some possible clearinghouse models, royalties may be set centrally on behalf of IP owners. To the extent that this overcomes the tragedy of the anticommons and lowers royalties, it will be beneficial. However, as we have 
seen, non-cooperative royalties may not be excessively high if IP rights are sufficiently substitutable. It would be interesting to use our framework to examine the effects of centralized royalty setting, in comparison with the decentralized outcome, and determine the implications of centralization for the welfare effects of a reduction in the costs of the licensing process. A framework similar to that used in this paper could be used to compare the four different types of clearinghouse that we identified in terms of effects on welfare, and the factors that affect the demand for the services of each type.

\section{Appendix}

\section{Proof of lemma 1}

If $r_{i}=r$ for all $i, z=n r^{\frac{\beta}{\beta-1}}$ and the downstream profitability constraint (4) is $r \leq(\alpha-2 \sqrt{s(n)}) / n^{\frac{\beta-1}{\beta}}$. We will show that any IP owner has an incentive to undercut any $r>0$ that satisfies this constraint, when $\beta>1 / n$. IP owner $i$ 's first-order condition is

$$
\frac{\partial R_{i}}{\partial r_{i}}=x_{i}+\frac{\partial x_{i}}{\partial r_{i}} r_{i}
$$

From (5) we have

$$
\frac{\partial x_{i}}{\partial r_{i}}=\frac{1}{\beta-1} r_{i}^{-\frac{\beta-2}{\beta-1}} z^{-\frac{1}{\beta}} q_{M}-\frac{1}{\beta} r_{i}^{\frac{1}{\beta-1}} z^{-\frac{\beta+1}{\beta}} \frac{\partial z}{\partial r_{i}} q_{M}+r_{i}^{\frac{1}{\beta-1}} z^{-\frac{1}{\beta}} \frac{\partial q_{M}}{\partial r_{i}}
$$

where

$$
\frac{\partial z}{\partial r_{i}}=\frac{\beta}{\beta-1} r_{i}^{\frac{1}{\beta-1}} \text { and } \frac{\partial q_{M}}{\partial r_{i}}=-\frac{1}{2} z^{-\frac{1}{\beta}} r_{i}^{\frac{1}{\beta-1}}
$$

Imposing symmetry, after some algebraic manipulation, we obtain

$$
\left.\frac{\partial R_{i}}{\partial r_{i}}\right|_{r_{i}=r \forall i}=\frac{n^{-\frac{\beta+1}{\beta}}(1-n \beta) \alpha}{2(1-\beta)}-\frac{n^{-\frac{2}{\beta}}(2-(1+n) \beta)}{2(1-\beta)} \times r
$$

Note this is a linear function of $r$. First, it is easy to verify that this is negative for all $r>0$ when $\beta \in(1 / n, 2 /(n+1))$ as the intercept and slope are both negative. For $\beta \in[2 /(n+1), 1]$, the intercept is negative, but the slope is positive. However, the maximum $r$ at which downstream production is profitable is $r=(\alpha-2 \sqrt{s(n)}) / n^{\frac{\beta-1}{\beta}}$. Substituting this into (14), $\left.\frac{\partial R_{i}}{\partial r_{i}}\right|_{r_{i}=r \forall i}$ is positive if

$$
\alpha<\frac{2(2-(1+n) \beta) \sqrt{s(n)}}{1-\beta}
$$


but the right-hand side of this expression is less than or equal to zero for $\beta \geq 2 /(n+1)$, while $\alpha$ is always positive, thus $\left.\frac{\partial R_{i}}{\partial r_{i}}\right|_{r_{i}=r \forall i}$ is negative for all $r \leq(\alpha-2 \sqrt{s(n)}) / n^{\frac{\beta-1}{\beta}}$.

\section{Proof of Proposition 1}

From lemma 1 we know that when $\beta>1 / n$, any symmetric royalty $r>0$ cannot be an equilibrium. If $r=0$ and $\beta>1 / n$, the manufacturer's cost minimization problem does not have a unique solution, but any level of output can be achieved at zero marginal cost using only a single input if necessary. Thus if all other licensors are setting zero royalties, no individual licensor can increase revenues by setting a strictly positive royalty, so $r=0$ is an equilibrium.

If $\beta \leq 1 / n$, ignoring the downstream profitability constraint for now, setting (14) equal to zero we obtain

$$
r=\frac{n^{\frac{1}{\beta}}\left(\frac{1}{n}-\beta\right) \alpha}{2-(1+n) \beta}
$$

It is straightforward but very tedious to verify that the second-order condition for a maximum of $R_{i}$ is satisfied by (15) when $\beta \leq 1 / n$, thus this value of $r$ satisfies the conditions for a symmetric Nash equilibrium. Substituting (15) into the profitability constraint (4) gives

$$
\alpha \geq \frac{2(2-(1+n) \beta)}{1-\beta} \sqrt{s(n)}
$$

If (16) does not hold, suppose that $r$ is such that profit is zero profit, i.e., (4) holds with equality. This gives

$$
r=n^{\frac{1-\beta}{\beta}}(\alpha-2 \sqrt{s(n)})
$$

which is necessarily lower than the value given by (15). At this $r$, from (14), $\left.\frac{\partial R_{i}}{\partial r_{i}}\right|_{r_{i}=r \forall i}$ is positive for when (16) holds, thus it is a symmetric Nash equilibrium as each IP owner cannot increase revenues by reducing $r_{i}$, and will cause the manufacturer to cease production if $r_{i}$ increases.

\section{Proof of Proposition 2}

The equilibrium royalty exceeds the joint optimum if $\rho>1$. First suppose $\bar{\alpha} \leq \alpha \leq A$. Then from (10), $\rho>1$ implies $2(1-n \beta)>2-(1+n) \beta$, or $\beta(1-n)>0$. If $\beta>0$ this is never true, while if $\beta<0$ it is true provided $n>1$. Thus equilibrium royalties for products in the range $\bar{\alpha} \leq \alpha \leq A$ never exceed the joint optimum if $\beta>0$, while if $\beta<0$ they equal the joint optimum if $n=1$ and exceed it if $n>1$. 
Now suppose that $\alpha \leq \alpha \leq \bar{\alpha}$. Then from (10), $\rho>1$ implies $\alpha>$ $4 \sqrt{s(n)}$. Recall that $\underline{\alpha}=2 \sqrt{s(n)}<4 \sqrt{s(n)}$. However, $\bar{\alpha}>4 \sqrt{s(n)}$ implies $\beta(1-n)>0$. This is true if $\beta<0$ and $n>1$, but not true if $\beta>0$. Thus $\rho>1$ is possible when $\beta<0, n>1$ and $4 \sqrt{s(n)}<\alpha \leq \bar{\alpha}$. But $\rho>1$ contradicts $\alpha \leq \bar{\alpha}$ when $\beta>0$, so $\rho>1$ is not possible for $\underline{\alpha} \leq \alpha \leq \bar{\alpha}$ when $\beta>0$.

\section{Proof of Proposition 5}

Using (8) and (7) to evaluate (11), after some manipulation we obtain

$$
T R=\frac{(1-n \beta)(1-\beta) A^{2}}{6(2-\beta(1+n))^{2}}+\frac{2(1-n \beta)((2-n) \beta-1) \sqrt{s(n)^{3}}}{3(1-\beta)^{2} A}
$$

Thus

$$
\frac{\partial T R}{\partial s(n)}=\frac{(1-n \beta)((2-n) \beta-1) \sqrt{s(n)}}{(1-\beta)^{2} A}
$$

Since $\beta \leq 1 / n, 1-n \beta \geq 0$. Thus $T R$ is a decreasing function of $s(n)$ if $(2-n) \beta>1$, otherwise it is an increasing function.

\section{Proof of Proposition 7}

Using (8) and (7) to evaluate (12), after some manipulation we obtain

$$
C S=\frac{(1-\beta)^{2} A^{2}}{24(2-\beta(1+n))^{2}}+\frac{(1-(2 n-1) \beta) \sqrt{s(n)^{3}}}{3(1-\beta) A}
$$

Thus

$$
\frac{\partial C S}{\partial s(n)}=\frac{(1-(2 n-1) \beta) \sqrt{s(n)}}{2(1-\beta) A}
$$

The sign of this is the same as the sign of $1-(2 n-1) \beta$. Thus if $1 /(2 n-1)<$ $\beta \leq 1 / n, C S$ is a strictly increasing function of $s(n)$, otherwise it is a decreasing function.

\section{Proof of Proposition 8}

Welfare in a given downstream market is $W(\alpha)=\alpha q-\frac{1}{2} q^{2}-s(n)$, thus total welfare is

$$
W=\int_{\underline{\alpha}}^{\bar{\alpha}} \frac{\alpha q_{M}\left(r^{*}(\alpha)\right)-\frac{1}{2} q_{M}\left(r^{*}(\alpha)\right)^{2}-s(n)}{A} d \alpha .
$$

Evaluating, after some manipulation we obtain

$$
\begin{aligned}
W= & \frac{(7-\beta(3+4 n))(1-\beta) A^{2}}{24(2-\beta(1+n))^{2}} \\
& +\frac{\left((2 n(n+1)+3) \beta^{2}-(8+6 n) \beta+7\right) \sqrt{s(n)^{3}}}{3 A(1-\beta)^{2}}-s(n)
\end{aligned}
$$


which can be written as $W(s(n))=a+b \sqrt{s(n)^{3}}-s(n)$. Welfare improves from a transaction cost reduction if $W\left(s_{1}(n)\right) \geq W\left(s_{0}(n)\right)$, which implies

$$
\frac{\sqrt{s_{0}(n)^{3}}-\sqrt{s_{1}(n)^{3}}}{s_{0}(n)-s_{1}(n)} \leq \frac{1}{b}
$$

The left-hand side of (20) is the slope of a ray between $\sqrt{s_{1}(n)^{3}}$ and $\sqrt{s_{0}(n)^{3}}$. Given $s_{0}(n)$, this slope decreases as $s_{1}(n)$ decreases, since $\sqrt{x^{3}}$ is a strictly convex function. Thus if (20) is satisfied for a marginal decrease in $s(n)$ from $s_{0}(n)$ it will also be satisfied for any larger decrease. This implies that if $\frac{3}{2} \sqrt{s_{0}(n)} \leq \frac{1}{b}$ then any transaction cost reduction will increase welfare, which requires

$$
s_{0}(n) \leq \frac{4 A^{2}(1-\beta)^{4}}{\left((2 n(n+1)+3) \beta^{2}-(8+6 n) \beta+7\right)^{2}} .
$$

Comparing this with (2), we see that any parameters that satisfy (2) also satisfy (21) if $\left(2 n^{2}-2 n-1\right) \beta^{2}-2 \beta(n-2)-1 \leq 0$. If $n=1$ this is true for any possible $\beta$. If $n \geq 2$, the coefficient on $\beta^{2}$ is positive and the roots of the quadratic in $\beta$ are

$$
\beta=\frac{n-2 \pm \sqrt{3}(n-1)}{2 n^{2}-2 n-1} .
$$

It is easily verified that the larger root exceeds $1 / n$ for $n \geq 2$, thus (21) always holds for any parameter values that satisfy (2) if $\beta$ is between the lower root and $1 / n$. Observe that this includes all values where $0<\beta \leq 1 / n$.

Otherwise, if $\beta<0$ and (21) does not hold, (20) may still hold if $s_{1}(n)$ is small enough. In such a case, for a given $s_{0}(n)$, if $b$ is larger, $s_{1}(n)$ must be smaller to satisfy (20). It is easily verified that $\frac{\partial b}{\partial n}>0$ and $\frac{\partial b}{\partial \beta}<0$.

\section{Proof of corollary 1}

From (19), it is clear that $W(0)>0$ and $W$ is decreasing in $s(n)$ at $s(n)=0$. In addition, it is easy to verify that the coefficient on $\sqrt{s(n)^{3}}$ is always positive. Thus $W$ is U-shaped in $s(n)$ and welfare does not increase for any transaction cost reduction if $W(0) \leq W\left(s_{0}(n)\right)$. This requires $s_{0}(n) \geq 1 / b^{2}$ where $b$ is the coefficient on $\sqrt{s(n)^{3}}$ in (19). In addition, the parameters must satisfy (2). After some rearrangement, there exist parameters that satisfy $(2)$ and $s_{0}(n) \geq 1 / b^{2}$ if $\left(4 n-2 n^{2}+3\right) \beta^{2}-10 \beta+5<$ 0 . This is never true for any $\beta<1 / n$ if $n=1$ or $n=2$. If $n \geq 3$, the coefficient on $\beta^{2}$ is negative and the roots in $\beta$ are

$$
\beta=\frac{-5 \pm \sqrt{10}(n-1)}{2 n^{2}-4 n-3} .
$$


The larger root exceeds $1 / n$, so there exist parameters where welfare does not increase for any transaction cost reduction if $\beta$ is less than the lower root.

\section{References}

[1] Aoki, R. (2006). Clearing houses and patent pools - Access to genetic patents. Department of Economics Working Paper \#264, University of Auckland.

[2] Aoki, R. \& S. Nagaoka (2005). Coalition formation for a consortium standard through a standard body and a patent pool: Theory and evidence from MPEG2, DVD and 3G. Institute of Innovation Research, Hitotsubashi University, Working Paper WP\#05-01.

[3] Aoki, R. \& A. Schiff (2008). Promoting access to intellectual property: Patent pools, copyright collectives and clearinghouses. RED Management, 38: 189-204.

[4] Arora, A. \& A. Fosfuri (2003). Licensing the market for technology. Journal of Economic Behavior and Organization, 52: 277-295.

[5] Arora, A., A. Fosfuri \& A. Gambardella (2001). Markets for Technology. MIT Press: Cambridge.

[6] Athreye, S. \& J. Cantwell (2007). Creating competition? Globalisation and the emergence of new technology producers. Research Policy, 36: 209-226.

[7] Besen, S., S. Kirby \& S. Salop (1992). An economic analysis of copyright collectives. Virginia Law Review, 78: 383-411.

[8] Buchanan, J. \& Y. Yoon (2000). Symmetric tragedies: Commons and anticommons. Journal of Law and Economics, 43: 1-13.

[9] Gallini, N. \& B. Wright (1990). Technology transfer under asymmetric information. RAND Journal of Economics, 21: 147-160.

[10] Graff, G. \& D. Zilberman (2001). Towards an intellectual property clearinghouse for ag-biotechnology: An issues paper. IP Strategy Today, 3: 1-17.

[11] Heller, M. \& R. Eisenberg (1998). Can patents deter innovation? The anticommons in biomedical research. Science, 280: 698-701.

[12] Hollander, A. (1984). Market structure and performance in intellectual property: The case of copyright collectives. International Journal of Industrial Organization, 2: 199-216. 
[13] Katz, M. \& C. Shapiro (1985). On the licensing of innovations. RAND Journal of Economics, 16: 504-520.

[14] Kleit, A. (2000). ASCAP versus BMI (versus CBS): Modeling competition between and bundling by performance rights organizations. Economic Inquiry, 38: 579-590.

[15] Lerner, J. \& J. Tirole (2004). Efficient patent pools. The American Economic Review, 94: 691-711.

[16] Merges, R. (1996). Contracting into liability rules: Intellectual property rights and collective rights organizations. California Law Review, 84: 1293-1393.

[17] OECD (2002). Genetic Inventions, Intellectual Property Rights and Licensing Practices: Evidence and Policies. Downloaded from www.oecd.org/dataoecd/42/21/2491084.pdf.

[18] Razgaitis, R. (2005). U.S./Canadian licensing in 2004: Survey results. Les Nouvelles, 35: 145-155.

[19] Shapiro, C. (2001). Navigating the patent thicket: Cross licenses, patent pools and standard setting. In Jaffe, E., Lerner, J. \& Stern, S., eds, Innovation Policy and the Economy, Volume I. MIT Press: Cambridge.

[20] Teece, D. (1977). Technology transfer by multinational firms: The resource cost of transferring technological know-how. The Economic Journal, 87: 242-261.

[21] Van Overwalle, G., E. van Zimmeren, B. Verbeure, G. Matthijs (2006). Models for facilitating access to patents on genetic innovations. Nature Reviews Genetics, 7: 143-154.

[22] Verbeure, B., E. van Zimmeren, G. Matthijs \& G. Van Overwalle (2006). Patent pools and diagnostic testing. TRENDS in Biotechnology, 24: 115-120.

[23] van Zimmeren, E., B. Verbeure, G. Matthijs \& G. Van Overwalle (2006). A clearing house for diagnostic testing: The solution to ensure access to and use of patented genetic innovations? Bulletin of the World Health Organization, 84: 352-359. 